\title{
Sealing Proximal Non- and Micro-Cavitated Carious Lesions Using a One-Session Separator Technique: A 2-Year Randomised Clinical Study
}

\author{
Birgitta Lindquist Claes-Göran Emilson \\ Department of Cariology, Institute of Odontology, Sahlgrenska Academy, University of Gothenburg, \\ Gothenburg, Sweden
}

\begin{abstract}
Keywords
Proximal caries diagnosis - Metal separator - Sealants .

Caries progression $\cdot$ Risk factors
\end{abstract}

\begin{abstract}
The presence of initial caries accounts for the majority of approximal carious lesions in many countries. The aim of this clustered, split-mouth, randomised, controlled clinical trial was to use a metal separator to widen the approximal space in a one-visit session before sealing and to evaluate patient acceptance, together with the efficacy of the proximal sealing in arresting incipient carious lesions after 2 years. A total of 48 patients with a mean age of 41.9 years were selected. They had at least one pair of proximal initial carious lesions, including the distal surface of the canines to the mesial surface of the third molars (bite-wing score D1-D3). The patient's caries risk at baseline was analyzed using a Cariogram. All surfaces were examined for mutans streptococci (ms) counts. The separator technique made it possible to diagnose whether or not a microcavity was present. After 2 years, 212 surfaces in 45 subjects were examined using the same as baseline standardized digital follow-up radiographs. Two analyses were performed, one sensitive, where a progression or a regression was assessed if one of the examiners made one of those diagnoses, and one conservative, where unchanged. For both analyses, the sealed test surfaces showed
\end{abstract}

karger@karger.com www.karger.com/cre

Karger $\stackrel{\text { ' }}{5}$

GOPEN ACCESS
(C) 2020 The Author(s)

Published by S. Karger AG, Basel

This article is licensed under the Creative Commons AttributionNonCommercial-NoDerivatives 4.0 International License (CC BYNC-ND) (http://www.karger.com/Services/OpenAccessLicense). Usage and distribution for commercial purposes as well as any distribution of modified material requires written permission. a significantly higher regression (67 and $29 \%$ ) compared to the control group ( 13 and $2 \%$ ) $p<0.0001$. It did not appear that the baseline variables, such as the caries risk, surface diagnoses, or ms counts, influenced the caries outcome. In the test group, there was no difference in caries progression if there was a microcavity or not. The separation treatment was well accepted by the patients. The method of separation for diagnosis and sealing treatment in a single session appears to be a clinically applicable method for the control of proximal carious lesions.

(C) 2020 The Author(s)

Published by S. Karger AG, Basel

\section{Introduction}

The prevalence of dental caries has declined in many countries during the last 5 decades, due mainly to the frequent use of different preventive measures, which have involved improved biofilm control, the increased frequent use of fluoride, and a focus on reducing the amount and frequency of sugar [Kidd et al., 2008; Frencken et al., 2017]. Despite the huge effort that is being made, untreated caries in permanent teeth constitutes the most prevalent disease in humans globally [Kassebaum et al., 2015].

Initial caries, which accounts for the majority of approximal carious lesions in many countries [Mejàre et al., 1998], may progress to a cavity, which needs to be treated 
with a restoration. This underlines the need for minimally invasive approaches to prevent or postpone the restorative treatment cycle [Qvist, 2008]. For approximal tooth surfaces, the use of resin sealing or infiltration as microinvasive treatment methods for initial non-cavitated carious lesions has shown promising clinical results when it comes to controlling the progression of approximal lesions in children and young adults, with various levels of caries risk [Gomez et al., 2005; Martignon et al., 2006, 2010, 2012; Meyer-Lueckel et al., 2012, 2016; Basili et al., 2017; Krois et al., 2018].

To make the approximal surface available for local treatment, the teeth in contact have to be separated, which also allows for an accurate diagnosis of the carious lesions. To facilitate approximal sealing, treatment with an orthodontic band has been used for separation. After 2-6 days, the band is removed, and the approximal surface is treated with $35 \%$ phosphoric acid and an adhesive sealing material [Gomez et al., 2005; Martignon et al., 2006, 2010]. However, this method requires a second visit by the patient, which is time-consuming. A one-visit procedure is used with the infiltration method. The surface of the lesion is treated with $15 \%$ hydrochloric acid, enabling the resin to penetrate the demineralized enamel in order to create a diffusion barrier inside the lesion [MeyerLueckel et al., 2007; Paris and Meyer-Lueckel, 2010]. However, the technique requires a rubber dam to protect the gingiva and the adjacent dental surface and a special commercial product for caries infiltration, which is expensive as it is a single-use product.

The general arguments against the use of approximal sealing and infiltration have been that they are complex and time-consuming [Splieth et al., 2010]. There is, therefore, a need for a one-visit, non-invasive method, which is practical and inexpensive. A special metal separator has been used to open up the space between neighboring teeth to enable a clinical diagnosis of the proximal carious lesions, which could be followed by a sealing procedure, all in a single session. The aim of the present study was to use this separator method and to evaluate its acceptance by adult patients, together with the efficacy of this sealing procedure for arresting incipient proximal carious lesions after 2 years.

\section{Materials and Methods}

The number of patients was based on the following premises: paired observations, $\alpha=5 \%, 1-\beta=80 \%$, clinically relevant effect not to be missed, $30 \%$, and effect of the least effective treatment, $25 \%$. Using the formula to test differences in proportions for the paired sample design [Connor, 1987], a sample size of about 40 patients was needed. During 2015 and 2016, patients (>20 years of age) at the Dental School, Gothenburg, were asked if they would like to participate in this prospective 2-year study. Prior to the invitation, a clinical examination including bite-wing radiographs was carried out by the students, together with their dental tutors.

The inclusion criterion was that each participant should have one or more pairs of radiographic proximal initial carious lesions (D1-3, radiolucency up to the outer third of the dentine). All lesions on the bitewing radiographs of each individual from the distal surface of the canines to the mesial surface of the third molar were recorded. They were randomly selected to the test or control group in each subject, using a random number table, without considering their intraoral location, radiographic diagnosis, or the tooth surface chosen. If the proximal lesion was planned to be included in any prosthetic treatment, it was excluded.

The caries risk was assessed individually at the clinic using the Cariogram software [Bratthall and Hänsel Petersson, 2005], which categorized the patient's risk as low (score $1=<40 \%$ ), medium (score $2=>40-60 \%)$ or high $($ score $3=>60 \%)$ [Petersson and Tvetman, 2015]. Salivary mutans streptococci and buffer capacity were estimated using selective chair-side kits (Dentocult ${ }^{\circledR}$ SM-Strip

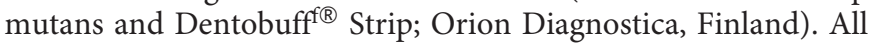
patients were included in the standard preventive program at the student clinic, including instructions on dental hygiene, diet, and the use of fluoride.

From the selected approximal lesions in the test and control groups, plaque samples were taken at the first treatment visit using the toothpick method for the presence of mutans streptococci (ms). This method has shown good reproducibility in repeated samplings and a remarkably low variation in recovery over a long period [Emilson et al., 1987; Lindquist and Emilson, 1990; Wennerholm et al., 1995]. Sampling was performed by inserting sterile wooden triangular toothpicks (TePe, Malmö, Sweden) into each interproximal space until firm contact was obtained. The side of the toothpick was pressed against the slightly convex surface of mitis salivarius bacitracin agar MSB [Gold et al., 1973]. The inoculated agar plates were incubated for 2 days at $37^{\circ} \mathrm{C}$ in $5 \% \mathrm{CO}_{2}$ in $\mathrm{N}_{2}$. Typical colonies of ms [Emilson, 1983] were counted. The presence of ms was determined for each tooth surface, and the number of colony-forming units (CFU) was counted and expressed in terms varying from 0 to 3 as follows: score $0=<5 \mathrm{CFU}$; score $1=5-10 \mathrm{CFU}$; score $2=11-100 \mathrm{CFU}$; and score $3=>100$ CFU.

To obtain access to the approximal space, tooth separation was performed. A metal separator (Ivory, Karl Schumacher ${ }^{\circledR}$; Southampton, PA, USA) was inserted into the approximal space and gently screwed in intervals until the space between the proximal surfaces was about $1 \mathrm{~mm}$, which was adequate for inspection and confirming the presence of the lesion (Fig. 1). Only visible examination without probing was performed to diagnose the presence of a microcavity or no microcavity (a microcavity is a small enamel surface disruption $[<1 \mathrm{~mm}]$ ). Cotton rolls were used to isolate the working area. The neighboring proximal tooth surface was protected with a metallic band and kept in place by the separator. The carious tooth area was cleaned and etched for $60 \mathrm{~s}$ with a $35 \%$ phosphoric acid gel (Ultra-Etch ${ }^{\circledR}$; DAB Dental AB, Sweden), thoroughly washed with water for $30 \mathrm{~s}$, and air-dried for $10 \mathrm{~s}$. When the approximal surface was completely dry, a light-cured, low-viscosity sealant (Clinpro Sealant ${ }^{\mathrm{TM}}$; 3 M Espe, Seefeld, Germany) was slow- 


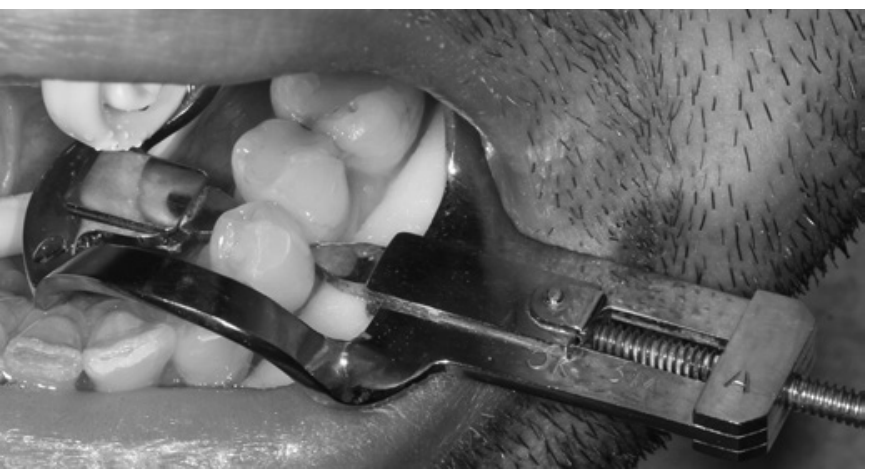

Fig. 1. Metal separator in place between the teeth.

ly applied in a very thin layer onto the etched area, following the manufacturer's instructions and according to an earlier study [Paris and Meyer-Lueckel, 2010]. Any excess material was removed using a Quickstick (Dentsolv ${ }^{\text {ab }}$; Saltsjö-Boo, Sweden). All sealants, which came from the same production batch, were light-

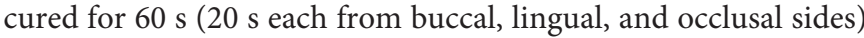
with a visible dental curing light (Curing Light XL 3000; 3 M ESPE). All contacts, visits, and treatments were performed by the same dentist (B.L.) and lasted for 15-25 min per treated surface.

After the sealing treatment, the participants were asked to describe their pain perception during the separation procedure. A modified visual analogue scale (VAS) was used to rate the intensity of their pain experience from 1 to 10 , where 1 represented a low sense of pain and 10 represented very high pain (unbearable, with a need for anesthesia). Out of consideration for the patients and for practical reasons, no tooth separation was performed in the control group of proximal lesions. Afterwards, the participants were told to visit their dental students for continued dental care as required. No restrictions were made for the selected lesions in future clinical treatments.

Two years later, the same follow-up standardized radiographic digital examination was performed. Two posterior bite-wing radiographs were taken from each side of the dentition (Focus FI04300; VistaScan, Dürr Dental, Tuusula, Finland). The central ray of one radiograph was directed perpendicular to the approximal space of the premolars and the other towards the first molar. If the approximal space of the test or control surfaces overlapped, a new radiograph was taken. Overlapping surfaces were recorded as not readable. The radiographs were coded and analyzed blindly by 2 external clinical observers from the Department of Cariology and Radiology at the institution working independently. As the sealing material is not radiopaque, the observers were unable to distinguish between test and control surfaces. There was no comparison and no discussion of their readings to reach a unified agreement. The inter-examiner agreement in diagnoses of the proximal surfaces was $67 \%$, weighted kappa of 0.55 with $95 \%$ CI (0.45-0.64) in the test, and in the control group the inter-examiner agreement was 56 and $78 \%$. One of the examiners gave higher scores for $44(20 \%)$ surfaces and the other examiner gave higher scores for $27(13 \%)$ surfaces $(p=0.057)$. No comparison of the intra-reproducibility of the radiological data was made.

The radiographic scoring system for the carious lesions was a slightly modified version of that suggested by Martignon et al.
[2006] as follows: D0 = radiographically sound (score 0); D1 = radiolucency in the outer half of the enamel (score 1); D2 = radiolucency from the inner half of the enamel including the enameldentine junction (score 2); D3 = radiolucency restricted to the outer third of the dentine (score 3 ). In the analysis 2 years later, a progression was defined when the final lesion score was higher than at baseline, while a regression was defined when a lower score was registered.

There was no comparison and no discussion of their readings to reach a unified agreement. Therefore, 2 analyses have been performed. In the first method of estimation (Sensitive Judgement), a progression or a regression were assessed if one of the examiners made one of those diagnosis, which means that a progression or a regression was registered if both examiners agreed, as well as if one observer had scored it as a progression or a regression. If the score 2 years later was the same, as noted by the 2 examiners, and similar to the baseline score, the surface was registered as stable and unchanged.

In the second more strict calculation (Conservative Judgement) only the unanimous diagnoses of regression or progression were included. If there was a disagreement upon the diagnosis of the surface, it was registered as stable and unchanged.

For comparison between the test and control surfaces, the Mantel-Haenszel $\chi^{2}$ exact test was used for ordered categorical variables and Fisher's exact test was used for dichotomous variables. Adjustment for tooth groups and caries diagnoses was made with the Mantel-Haenszel pooling technique applied to the Mantel-Haenszel $\chi^{2}$ test and $\chi^{2}$ test. These analyses were not adjusted for the dependence within subjects. Generalized estimation equations regression analyses with link $=$ logit were performed to adjust the analyses of regression and progression between the 2 groups for the dependence within subjects. These results are given as an adjusted odds ratio (OR) with $95 \%$ confidence intervals (CIs). The inter-reproducibility of the 2 radiographic observers was given with percentage agreement and weighted kappa, together with the sign test to analyze systematic differences. All significance tests were two-sided and conducted at the 5\% significance level.

\section{Results}

A total of 48 patients participated at baseline, but as 3 individuals could not be reached for the follow-up examination 2 years later, the final number of participants was 45 . The included subjects ( 22 females and 23 males) had a mean age of 41.9 years (range 26-65). The patients had one to 6 pairs of test and control surfaces. The Cariogram showed that the majority of participants had a high caries risk ( $n=23 ;>60 \%$; score 3$)$, while 14 had a medium caries risk (40-60\%; score 2), and 8 had a low caries risk ( $<40 \%$; score 1$)$, with a mean score of 2.3 (SD $0.77)$. The highest ms score was found on surfaces from patients with a high caries risk (test surfaces $81 \%$ and control sites $69 \% ; p=0.067$ ).

In the test group, lesions were located on 5 canines, 84 premolars, and 16 molars. The corresponding numbers 
Table 1. Distribution of tooth groups, caries risk, bacterial score, caries diagnosis, and progression in the test and the control surfaces

\begin{tabular}{|c|c|c|c|}
\hline Variable & $\begin{array}{l}\text { Test surfaces } \\
(n=105)\end{array}$ & $\begin{array}{l}\text { Control } \\
\text { surfaces } \\
(n=107)\end{array}$ & $p$ value \\
\hline \multicolumn{4}{|l|}{ Tooth groups } \\
\hline Canines & $5(5)$ & $9(8)$ & \\
\hline Premolars & $84(80)$ & $45(42)$ & \\
\hline Molars & $16(15)$ & $53(50)$ & $<0.001$ \\
\hline \multicolumn{4}{|l|}{ Caries risk score } \\
\hline$<40 \%$ & $25(24)$ & $25(23)$ & \\
\hline$>40-60 \%$ & $25(24)$ & $25(23)$ & \\
\hline$>60 \%$ & $55(52)$ & $57(54)$ & 0.90 \\
\hline \multicolumn{4}{|l|}{ Bacterial score } \\
\hline $\mathrm{CFU} \leq 5$ & $27(26)$ & $23(21)$ & \\
\hline$>5-10$ & $18(17)$ & $24(22)$ & \\
\hline$>10-100$ & $29(28)$ & $28(26)$ & \\
\hline$>100$ & $31(30)$ & $32(30)$ & 0.82 \\
\hline \multicolumn{4}{|l|}{ Caries diagnosis ${ }^{\mathrm{a}}$} \\
\hline D1 & $67(64)$ & $75(70)$ & \\
\hline D2 & $36(34)$ & $30(28)$ & \\
\hline D3 & $2(2)$ & $2(2)$ & 0.37 \\
\hline \multicolumn{4}{|c|}{ Main outcome lesion change sensitive judgements ${ }^{\mathrm{b}}$} \\
\hline Regression & $70(67)$ & $14(13)$ & \\
\hline Unchanged & $23(22)$ & $51(48)$ & \\
\hline Progression & $12(11)$ & $42(39)$ & $\begin{array}{l}<0.0001 \\
<0.0001\end{array}$ \\
\hline \multicolumn{4}{|c|}{ Main outcome lesion change conservative judgements ${ }^{c}$} \\
\hline Regression & $30(28)$ & $2(2)$ & \\
\hline Unchanged & $69(66)$ & $75(70)$ & \\
\hline Progression & $6(6)$ & $30(28)$ & $\begin{array}{l}<0.0001 \\
<0.0001\end{array}$ \\
\hline
\end{tabular}

For categorical variables $n(\%)$ is presented. For comparison between groups, the Mantel-Haenszel $\chi^{2}$ exact test was used for ordered categorical variables. $p$ value for comparison between test and control surfaces, not adjusted for individual dependence. ${ }^{\text {a }} \mathrm{D} 1$ = lesion to half of the enamel; D2 = lesion to the enamel-dentine border; D3 = lesion into third of the dentine. ${ }^{\mathrm{b}}$ Sensitive judgements $=$ progression or regression, if one of the examiners made those diagnoses. ${ }^{c}$ Conservative judgements $=$ the agreement of progression and regression, otherwise unchanged. ${ }^{\mathrm{d}} p$ value adjusted for tooth groups. Bold values are significant.

for the control surfaces were 9 canines, 45 premolars, and 53 molars. There was a significant difference between the groups of teeth belonging to the test and the control group (Table 1). Both the test and control lesions were distributed in about the same way in both jaws, and most of the lesions were diagnosed as D1 in both groups.

A total of 212 approximal surfaces were examined after 2 years in 2017 and 2018. As 2 test surfaces could not be diagnosed at the follow-up, a total of 105 test lesions and
107 control sites were available for radiographic evaluation and comparison.

\section{Sensitive Judgement}

In the test group, $67 \%$ of the surfaces showed regression after 2 years, compared with $13 \%$ in the control surfaces (Table 1). The difference of $54 \%$ was statistically significant, with an OR of 13.4 with 95\% CI (6.24-28.6), $p<$ 0.0001 , adjusted for tooth group. Only $11 \%$ showed progression in the test group compared with $39 \%$ of the control surfaces, with a mean difference of $28 \%$ and with an adjusted OR of 0.17 with $95 \% \mathrm{CI}(0.076-0.36), p<0.0001$, adjusted for tooth group. When comparing the tooth group of premolars, 55 (65\%) of the test surfaces showed regression compared with 7 (18\%) of the control surfaces, $p<0.0001$. A value of $18 \%$ indicated that the sealed surfaces had lower risk to progress than untreated surfaces and that the sealing induced a $82 \%$ decrease in the risk of caries progression.

\section{Conservative Judgement}

In the test group, $28 \%$ of the surfaces showed regression after 2 years, compared with $2 \%$ in the control surfaces (Table 1). The difference of $26 \%$ was statistically significant, with an OR of 14.0 with 95\% CI (3.16-62.0), $p<$ 0.0001 , adjusted for tooth group. Only $6 \%$ showed progression in the test group compared with $28 \%$ of the control surfaces, with a mean difference of $22 \%$ and with an adjusted OR of 0.13 with $95 \%$ CI (0.05-0.35), $p<0.0001$, adjusted for tooth group.

Since no differences were found for caries risk, the size score of the lesion or the amount of cariogenic bacteria between the test surfaces and the control surfaces (Table $1)$, no adjustment for these variables was needed.

After separation, the test lesions were inspected, and the existence or non-existence of a microcavity could be observed. The number of lesions with a microcavity was 49 (47\%), while the remainder without totalized 56 (53\%; Table 2). No separation was made in the control group.

When using the Sensitive Judgement, 37 (66\%) of the test surfaces without a cavity showed a regression compared with $33(67.3 \%)$ in the microcavitated group with a difference of $1.2 \%(p=1.00)$, with an OR of $1.0295 \%$ CI (0.43-2.41), $p=0.96$, adjusted for caries diagnosis. The corresponding values of regression in the Conservative Judgement was $10(17.9 \%)$ for test surfaces without a microcavity and $20(40.8 \%)$ for those showing a microcavity, with a difference of $22.9 \%$ ( $p=0.016$, adjusted diagnosis group, $p=0.049$ ), adjusted OR diagnosis group $0.3995 \%$ CI $(0.15-1.0), p=0.0500$. 
Table 2. Distribution of tooth groups, caries risk, bacterial score, caries diagnosis, and progression in the test surfaces group with or without a microcavity

\begin{tabular}{|c|c|c|c|}
\hline Variable & $\begin{array}{l}\text { Without } \\
\text { microcavity } \\
(n=56)\end{array}$ & $\begin{array}{l}\text { With } \\
\text { microcavity } \\
(n=49)\end{array}$ & $p$ value \\
\hline \multicolumn{4}{|l|}{ Tooth groups } \\
\hline Canines & $4(7)$ & $1(2)$ & \\
\hline Premolars & $42(75)$ & $42(86)$ & \\
\hline Molars & $10(18)$ & $6(12)$ & 0.95 \\
\hline \multicolumn{4}{|l|}{ Caries risk score } \\
\hline$<40 \%$ & $16(29)$ & $9(18)$ & \\
\hline$>40-60 \%$ & $12(21)$ & $13(27)$ & \\
\hline$>60 \%$ & $28(50)$ & $27(55)$ & 0.34 \\
\hline \multicolumn{4}{|l|}{ Bacterial score } \\
\hline $\mathrm{CFU} \leq 5$ & $17(30)$ & $10(20)$ & \\
\hline$>5-10$ & $8(14)$ & $10(20)$ & \\
\hline$>10-100$ & $14(25)$ & $15(31)$ & \\
\hline$>100$ & $17(30)$ & $14(29)$ & 0.60 \\
\hline \multicolumn{4}{|l|}{ Caries diagnosis $^{\mathrm{a}}$} \\
\hline D1 & $44(79)$ & $23(47)$ & \\
\hline D2 & $12(21)$ & $24(49)$ & \\
\hline D3 & $0(0)$ & $2(4)$ & $<0.001$ \\
\hline \multicolumn{4}{|c|}{ Main outcome lesion change sensitive judgements ${ }^{\mathrm{b}}$} \\
\hline Regression & $37(66)$ & $33(67)$ & \\
\hline Unchanged & $14(25)$ & $9(18)$ & \\
\hline Progression & $5(9)$ & $7(14)$ & $\begin{array}{l}0.76 \\
0.79^{d}\end{array}$ \\
\hline \multicolumn{4}{|c|}{ Main outcome lesion change conservative Judgements ${ }^{c}$} \\
\hline Regression & $10(18)$ & $20(41)$ & \\
\hline Unchanged & $45(80)$ & $24(49)$ & \\
\hline Progression & $1(2)$ & $5(10)$ & $\begin{array}{l}0.17 \\
0.27^{d}\end{array}$ \\
\hline
\end{tabular}

For categorical variables $n(\%)$ is presented. For comparison between groups, the Mantel-Haenszel $\chi^{2}$ exact test was used for ordered categorical variables. Adjustment for caries diagnosis was performed with Mantel-Haenszel pooling. $p$ value for comparison between surfaces with or without microcavity, not adjusted for individual dependence. ${ }^{\text {a }}$ D1 = lesion to half of the enamel; D2 = lesion to the enamel-dentine border; D3 = lesion into outer third of the dentine. ${ }^{\mathrm{b}}$ Sensitive judgements $=$ progression or regression, if one of the examiners made those diagnoses. ${ }^{c}$ Conservative judgements = the agreement of progression and regression, otherwise unchanged. ${ }^{\mathrm{d}} p$ value adjusted for caries diagnosis. The bold value is significant.

The separation treatment did not lead to any observed enamel surface breakdown. The separation with the separator instrument was well tolerated with basically, no pain or discomfort. No patient required local anesthesia or discontinued the treatment. Most of the assessments showed VAS scores of $1-5$, and only 13 subjects (29\%) indicated a moderate pain perception from 6 to 8 (Fig. 2).

Sealing Proximal Lesions at One Session

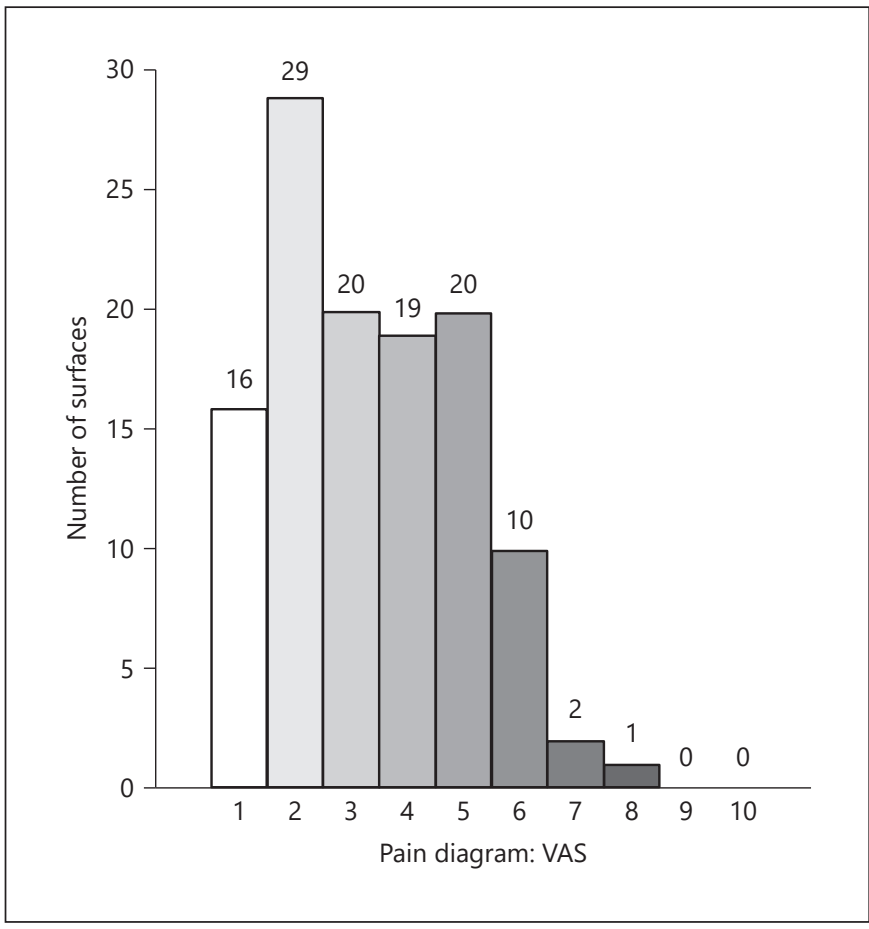

Fig. 2. Pain perception during the separation procedure (117 sessions in 48 patients at baseline).

\section{Discussion}

In this study, we used a metal separator, which has been used clinically for many years by the dentists at our department for the proximal surface inspection of caries diagnosed by radiographic examination which makes it possible to distinguish between a manifest cavitation or no cavitation [Mejàre et al., 1998]. Using this method for sealing, we were able to treat the carious proximal site in only one visit, as is done with the infiltration technique. The advantage of the present technique is that there is no need for a special instrument (separator combined with a matrix), which is expensive. In this study, no rubber dam was required.

In the study by Martignon et al. [2012], the infiltration technique was compared with sealing and placebo. Even though they found a greater therapeutic effect using infiltration, there was no significant difference between the 2 methods after 3 years. They made an interesting observation relating to enamel lesions reaching into the outer third of the dentine, where the sealed carious surfaces progressed less compared with the infiltrated teeth. The authors suggested that the use of hydrochloric acid for etching, which significantly reduces the thickness of the 
protective enamel surface layer compared with phosphoric acid, could be a possible reason for the difference.

The general arguments against the use of approximal sealing have been that the method is as complex to comply with and as time-consuming as approximal fillings [Splieth et al., 2010]. The one-visit sealing treatment used in this study could be performed with a chair time of 15$25 \mathrm{~min}$. The separation technique was well accepted by the participants. No complaints or undesirable side effects due to the treatment were reported, which is in agreement with other studies and their separation techniques [Ekstrand et al., 2010; Martignon et al., 2010; Alkilzy et al., 2011].

In this study, sealed proximal lesions showed a high prevalence of regression after 2 years, which has to be regarded as clinically successful, which was significantly greater compared with the untreated lesions. The therapeutic approach of sealing demineralized approximal surfaces is in accordance with the concept of minimally invasive dentistry [Ericson et al., 2003].

In general, the sealing or infiltration studies have not shown the results for caries regression, but in some studies have also presented reversals of test proximal surfaces [Martignon et al., 2006; Alkilzy et al., 2009, 2011; Trairatvorakul et al., 2011].

In our study, $12 \%$ of the sealed approximal surfaces showed a progression of the lesions compared with $41 \%$ in the untreated group. This progression rate for the test surfaces was lower compared with earlier studies of primary and permanent teeth, where frequency figures between 16\% [Abuchaim et al., 2010] and 31-40\% have been observed [Ekstrand et al., 2010, Martignon et al., 2012]. This could be due to younger participants being included in these studies.

The visual inspection after tooth separation before sealing may serve as a diagnostic tool to supplement conventional and radiographic examination [Hintze et al., 1998]. This could be of clinical importance, as earlier studies have shown that cavitated carious lesions are thought to be prone to further progression and therefore indicative of restorative treatment [Kidd and Pitts, 1990]. In our study, we also examined the approximal test surfaces for the presence of a small enamel surface disruption (microcavity). However, the existence of a microcavity did not influence the progression of the lesions. Moreover, the general caries risk, the prevalence of $\mathrm{ms}$ or the caries diagnosis (score 1-3) of the treated lesions at baseline did not appear to influence the caries progression. This was somewhat unexpected, since ms are bacteria most commonly associated with initiation of dental caries, and a higher caries diagnosis is correlated to a caries progression and has been used in earlier studies [Mejàre et al., 1998; Gomez et al., 2014; Ghazal et al., 2018]. The caries risk at baseline might have been reduced due to intensive prophylaxis by the students during the 2 years of the study.

The same dentist performed all sealings of the proximal surfaces and knew the distribution of the test and control surfaces. All radiographic readings were made by 2 examiners who had long experience of the radiographic diagnosis of carious lesions. They were blinded with regard to test or control lesions, as the resin-sealed lesions were optically masked in the radiographs, as the refractive index is close to that of the enamel.

There are some limitations to this study. First, conventional readings of the radiograph were used to examine the progression of the lesions at baseline and 2 years later. No digital subtraction radiography, a method which is considered to be a more sensitive technique than conventional readings of radiographs, was available [Ricketts et al., 2007]. However, Martignon et al. [2012] found no differences between conventional radiographs and digital subtraction radiography. Second, the baseline and follow-up radiographs were taken by different health-care providers. No individualized full-arch bitewing holders allowing the more exact alignment of the X-ray tube-jawfilm system were used, which means a lack of angulation. Third, the radiographs were examined separately by the 2 dentists, and there was no discussion between them to reach a unified decision. This may be the reason for the inter-examiner reliability value of $67 \%$ (weighted kappa; 0.55 ), a value which shows good reproducibility but is lower than in other studies [Alkilzy et al., 2009, 2011]. However, there was no indication of systematic inter-examiner deviations. This could also be the reason for the higher regression score in our study compared with other studies in this area, but the result when using this method could have been the opposite. Another limitation is that we decided out of consideration for the patients and for practical reasons, that no tooth separation should be performed in the control group of proximal lesions. This means that we are unable to compare the outcome between the existence of a microcavity between the test and control groups. However, there was no indication of any such influence when comparing test surfaces with or without microcavities. A further limitation was that the number of molars in the control group was higher than that in the test group (53 vs. 16), even though a random table was used to select the test and control lesions. This may have influenced the outcome, as the proximal surfaces of molars are expected to have a wider contact area 
compared with premolars, leading to a higher caries risk [Mejàre et al., 1999].

To conclude, the results of the present study show that this carious sealing method using a temporary separator is a clinically applicable interceptive method for the treatment of interproximal carious lesions. This one-visit, non-invasive method can be performed with reasonable chair time in the absence of anesthesia, is inexpensive, and may conserve tooth structures by delaying intervention or minimizing the restorative procedure.

\section{Acknowledgement}

The authors would like to thank Ann-Britt Lundberg for her laboratory assistance, together with Cecilia Johansson and Annika Ekestubbe, for their radiographic examinations. Nils-Gunnar Pehrsson is acknowledged for assisting with the statistical analysis. We gratefully extend our thanks to the personnel in the Public Health Service at the student clinic.

\section{Statement of Ethics}

Ethical approval was obtained from the local ethical board at the University of Gothenburg (Dnr:007-15). The authors have adhered to the Declaration of Helsinki. Volunteers gave their written informed consent to the study protocol, which was approved by the University of Gothenburg, as well as the community dental care service.

\section{Disclosure Statement}

The authors declare no conflicts of interest with respect to the authorship and/or publication of this article.

\section{Funding Sources}

This study was supported by grants from the Swedish Patent Revenue Fund for Research in Preventive Odontology (Dnr:2015006). The sealing material was provided by $3 \mathrm{M}$ Espe. The funders had no role in the study design, data collection and analysis, decision to publish, or preparation of the manuscript.

\section{Author Contributions}

B.L. contributed to the conception, design, data acquisition, analysis and interpretation, and drafted and critically revised the manuscript; C.-G.E. contributed to the conception, design, and interpretation and critically revised the manuscript. Both authors gave their final approval and agree to be accountable for all aspects of the work.

\section{References}

Abuchaim C, Rotta M, Grande RH, Loguercio $\mathrm{AD}$, Reis A. Effectiveness of sealing active proximal caries lesions with an adhesive system: 1 -year clinical evaluation. Braz Oral Res. 2010 Jul-Sep;24(3):361-7.

Alkilzy M, Berndt C, Meller C, Schidlowski M, Splieth C. Sealing of proximal surfaces with polyurethane tape: a two-year clinical and radiographic feasibility study. J Adhes Dent. 2009 Apr;11(2):91-4.

Alkilzy M, Berndt C, Splieth CH. Sealing proximal surfaces with polyurethane tape: threeyear evaluation. Clin Oral Investig. 2011 Dec; 15(6):879-84

Basili CP, Emilson CG, Corvalan GC, Moran MP Torres C, Quiroz MD, et al. Preventive and therapeutic proximal sealing: a 3.5-year randomized controlled clinical trial follow-up. Caries Res. 2017;51(4):387-93.

Bratthall D, Hänsel Petersson G. Cariogram-a multifactorial risk assessment model for a multifactorial disease. Community Dent Oral Epidemiol. 2005 Aug;33(4):256-64.

Connor RJ. Sample size for testing differences in proportions for the paired-sample design. Biometrics. 1987 Mar;43(1):207-11.
Ekstrand KR, Bakhshandeh A, Martignon S. Treatment of proximal superficial caries lesions on primary molar teeth with resin infiltration and fluoride varnish versus fluoride varnish only: efficacy after 1 year. Caries Res. 2010;44(1):41-6.

Emilson CG, Lindquist B, Wennerholm K. Recolonization of human tooth surfaces by Streptococcus mutans after suppression by chlorhexidine treatment. J Dent Res. 1987 Sep;66(9):1503-8.

Emilson CG. Prevalence of Streptococcus mutans with different colonial morphologies in human plaque and saliva. Scand J Dent Res. 1983 Feb;91(1):26-32.

Ericson D, Kidd E, McComb D, Mjör I, Noack MJ. Minimally Invasive Dentistry-concepts and techniques in cariology. Oral Health Prev Dent. 2003;1(1):59-72.

Frencken JE, Sharma P, Stenhouse L, Green D, Laverty D, Dietrich T. Global epidemiology of dental caries and severe periodontitis - a comprehensive review. J Clin Periodontol. 2017 Mar;44 Suppl 18:S94-105.

Ghazal TS, Levy SM, Childers NK, Carter KD, Caplan DJ, Warren JJ, et al. Mutans streptococci and dental caries: A new statistical modelling approach. Caries Res. 2018;52(3):246-52.
Gold OG, Jordan HV, Van Houte J. A selective medium for Streptococcus mutans. Arch Oral Biol. 1973 Nov; 18(11):1357-64.

Gomez SS, Basili CP, Emilson CG. A 2-year clinical evaluation of sealed noncavitated approximal posterior carious lesions in adolescents. Clin Oral Investig. 2005 Dec;9(4):239-43.

Gomez SS, Emilson CG, Corvalan GC, Quiroz MD, Moran MP. Efficacy of sealing the mesial surfaces of first permanent molars with respect to the status of the distal surfaces of the second primary molars in children at high caries-risk. Eur Arch Paediatr Dent. 2014 Apr;15(2):65-73.

Hintze H, Wenzel A, Danielsen B, Nyvad B. Reliability of visual examination, fibre-optic transillumination, and bite-wing radiography, and reproducibility of direct visual examination following tooth separation for the identification of cavitated carious lesions in contacting approximal surfaces. Caries Res. 1998;32(3):204-9.

Kassebaum NJ, Bernabé E, Dahiya M, Bhandari B, Murray CJ, Marcenes W. Global burden of untreated caries: a systematic review and metaregression. J Dent Res. 2015 May;94(5): $650-8$. 
Kidd EA, Pitts NB. Temporary elective tooth separation as a diagnostic aid in general practice. Br Dent J. 1990;169:195-200.

Kidd EA, van Amerongen JP, van Amerongen WE. The role of operative treatment in caries control; Fejerskov O, Kidd EAM (eds): Dental Caries: The disease and its clinical management. Oxford, Blackwell Munksgaard. 2008. pp. 355-65.

Krois J, Göstemeyer G, Reda S, Schwendicke F. Sealing or infiltrating proximal carious lesions. J Dent. 2018 Jul;74:15-22.

Lindquist B, Emilson CG. Distribution and prevalence of mutans streptococci in the human dentition. J Dent Res. 1990 May;69(5):11606.

Martignon S, Ekstrand KR, Ellwood R. Efficacy of sealing proximal early active lesions: an 18-month clinical study evaluated by conventional and subtraction radiography. Caries Res. 2006;40(5):382-8.

Martignon S, Ekstrand KR, Gomez J, Lara JS, Cortes A. Infiltrating/sealing proximal caries lesions: a 3-year randomized clinical trial. J Dent Res. 2012 Mar;91(3):288-92.

Martignon S, Tellez M, Santamaría RM, Gomez J, Ekstrand KR. Sealing distal proximal caries lesions in first primary molars: efficacy after 2.5 years. Caries Res. 2010;44(6):562-70.
Mejàre I, Källestål C, Stenlund H. Incidence and progression of approximal caries from 11 to 22 years of age in Sweden: A prospective radiographic study. Caries Res. 1999;33(2):93100.

Mejàre I, Källestål C, Stenlund H, Johansson H. Caries development from 11 to 22 years of age: a prospective radiographic study. Prevalence and distribution. Caries Res. 1998;32(1): 10-6.

Meyer-Lueckel H, Balbach A, Schikowsky C, Bitter K, Paris S. Pragmatic RCT on the efficacy of proximal caries infiltration. J Dent Res. 2016 May;95(5):531-6.

Meyer-Lueckel H, Bitter K, Paris S. Randomized controlled clinical trial on proximal caries infiltration: three-year follow-up. Caries Res. 2012;46(6):544-8.

Meyer-Lueckel H, Paris S, Kielbassa AM. Surface layer erosion of natural caries lesions with phosphoric and hydrochloric acid gels in preparation for resin infiltration. Caries Res. 2007;41(3):223-30

Paris S, Meyer-Lueckel H. Inhibition of caries progression by resin infiltration in situ. Caries Res. 2010;44(1):47-54.
Petersson GH, Twetman S. Caries risk assessment in young adults: a 3 year validation of the Cariogram model. BMC Oral Health. 2015 Jan; 15(1):17.

Qvist V. Longevity of restorations: the 'death spi-

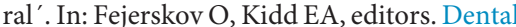
caries: the disease and its clinical management. Oxford, UK: Blackwell Munksgaard; 2008. pp. 444-55.

Ricketts DN, Ekstrand KR, Martignon S, Ellwood $R$, Alatsaris M, Nugent Z. Accuracy and reproducibility of conventional radiographic assessment and subtraction radiography in detecting demineralization in occlusal surfaces. Caries Res. 2007;41(2):121-8.

Splieth CH, Ekstrand KR, Alkilzy M, Clarkson J, Meyer-Lueckel H, Martignon S, et al. Sealants in dentistry: outcomes of the ORCA Saturday afternoon symposium 2007. Caries Res. 2010; 44(1):3-13.

Trairatvorakul C, Itsaraviriyakul S, Wiboonchan W. Effect of glass-ionomer cement on the progression of proximal caries. J Dent Res. 2011 Jan;90(1):99-103.

Wennerholm K, Lindquist B, Emilson CG. The toothpick method in relation to other plaque sampling techniques for evaluating mutans streptococci. Eur J Oral Sci. 1995 Feb;103(1): $36-41$. 